\title{
Semiconductor neutron detectors
}

\section{R. Mendicino ${ }^{* a, b}$, A. Bagolini' ${ }^{c}$, M. Boscardin ${ }^{c, b}$, G.-F. Dalla Betta ${ }^{a, b}$, M. Dalla} Palma $^{a, d}$, A. Quaranta ${ }^{a, d}$

${ }^{a}$ Department of Industrial Engineering, University of Trento, Via Sommarive 9, 38123 Trento, Italy

${ }^{b}$ INFN TIFPA,

Via Sommarive 14, 38123 Trento, Italy

${ }^{c}$ Fondazione Bruno Kessler (FBK-CMM)

Via Sommarive 18, 38123 Trento, Italy

${ }^{d}$ INFN LNL,

Viale dell'Universitá, 2, 35020, Legnaro, Italy

$\dagger$

E-mail: roberto.mendicinodunitn.it

Semiconductor neutron detectors are attracting increasing interest in many application fields, due to the shortage and high cost of ${ }^{3} \mathrm{He}$ gas which represented for decades the most important solution to neutron detection. The most interesting devices consist of silicon sensors featuring high aspect-ratio cavities filled with neutron converter materials (typically based on ${ }^{10} \mathrm{~B}$ or ${ }^{6} \mathrm{Li}$ ). Very good results have so far been obtained by different research groups in Europe, but most of all in the USA, with efficiency values up to $50 \%$ using only a single detector layer. In this paper, the state of the art in semiconductor neutron detectors will be reviewed, with emphasis on silicon based devices. Moreover, recent results from the R\&D activity carried out in the framework of the INFN HYDE (HYbrid DEtector for neutrons) Project will be reported, covering design and technological aspects, as well as simulation results relevant to a new pixel sensor structure, aimed at high detection efficiency while minimizing the process complexity.

24th International Workshop on Vertex Detectors - VERTEX2015-

1-5 June 2015

Santa Fe, New Mexico, USA

\footnotetext{
* Speaker.

${ }^{\dagger}$ This work was supported in part by the Italian National Institute for Nuclear Physics (INFN) through the CSN5 project HYDE, and by the Provincia Autonoma di Trento through the Project MEMS2.
} 


\section{Introduction}

Semiconductor neutron detectors have many applications in different fields, such as security, medical imaging, cultural heritage, forensics, and high energy and nuclear physics, to cite a few. The shortage and the consequent increasing cost of ${ }^{3} \mathrm{He}$ gas, on which most neutron detector systems were based for decades, has played an important role in boosting the development of silicon based devices as detectors of thermal neutrons (see e.g. [1] and references therein). However, the cross section for thermal neutrons in silicon is very low (in the order of 2.24 barns) [2] and for this reason generally the neutron interactions are detected as an indirect effect. The two materials that are commonly used as a converter are ${ }^{10} \mathrm{~B}$ and ${ }^{6} \mathrm{Li}$, featuring a relative high cross section of 3840 barn and 940 barn, respectively. The Boron reaction with neutron is the following:

$$
{ }^{10} B+n\left\{\begin{array}{l}
\stackrel{94 \%}{\stackrel{6}{\longmapsto}} \alpha(1.47 \mathrm{MeV})+{ }^{7} \mathrm{Li}(0.84 \mathrm{MeV})+\gamma(0.48 \mathrm{MeV}) \\
\stackrel{6 \%}{\longmapsto} \alpha(1.78 \mathrm{MeV})+{ }^{7} \mathrm{Li}(1.01 \mathrm{MeV})
\end{array}\right.
$$

When a thermal neutron is absorbed the most probable reaction will produce ${ }^{7} \mathrm{Li}$ at $0.84 \mathrm{MeV}$ in its first excited state that rapidly decades to the ground state releasing $480 \mathrm{keV}$ gamma and alpha at $1.47 \mathrm{MeV}$. The other possibility is the release of ${ }^{7} \mathrm{Li}$ at the ground state with an energy of 1.01 $\mathrm{MeV}$ and an alpha particle at $1.78 \mathrm{MeV}$, the two different particles being released in opposition directions.

The Lithium reaction with neutron is the following:

$$
{ }^{6} \mathrm{Li}+n \longmapsto{ }^{3} \mathrm{H}(2.72 \mathrm{MeV})+\alpha(2.05 \mathrm{MeV})
$$

In the case of ${ }^{6} \mathrm{Li}$ the reaction products have higher energy and longer penetrating range compared to the reaction of Boron. As shown in Fig.1 there are two different approaches to solid state neutron detectors [2]. The first approach regards neutron-sensitive materials (e.g., $\mathrm{LinSe}_{2}$ ), where most of the charge from the particle reaction is available for transduction within the sensor itself [3]. As a result, the efficiency can be very high, but this approach is very difficult: the main problem is the crystal quality and the understanding of defects that significantly reduce the signal charge collection [4].

The second approach makes us of semiconductor sensors coated with thin films of neutron converter materials: the mean free path of the neutron reaction products should be long enough to penetrate in the semiconductor sensor depleted region, where they induce a signal by ionization. In this case the sensor material is usually silicon[5].

The simplest example for this type of detector consists of silicon planar sensors coated with a thin film of converter material (e.g., ${ }^{10} \mathrm{~B}$ or ${ }^{6} \mathrm{Li}$ ). In this case only one of the two particles outcoming from the neutron reaction can be detected. Most of all, an intrinsic trade-off regards the thickness of the converter layer: thicker layers would increase the probability of neutron conversion, but at the same time they would decrease the probability for reaction products to reach the silicon sensor. As a result, the typical efficiency for this type of configuration is limited to small values in the range $2 \%-5 \%[6]$.

In the last decade, in order to optimize the efficiency, differents solutions have been developed. The first solution was to have stack of planar sensors coated with ${ }^{6} \mathrm{LiF}$ [5]. A big improvement 

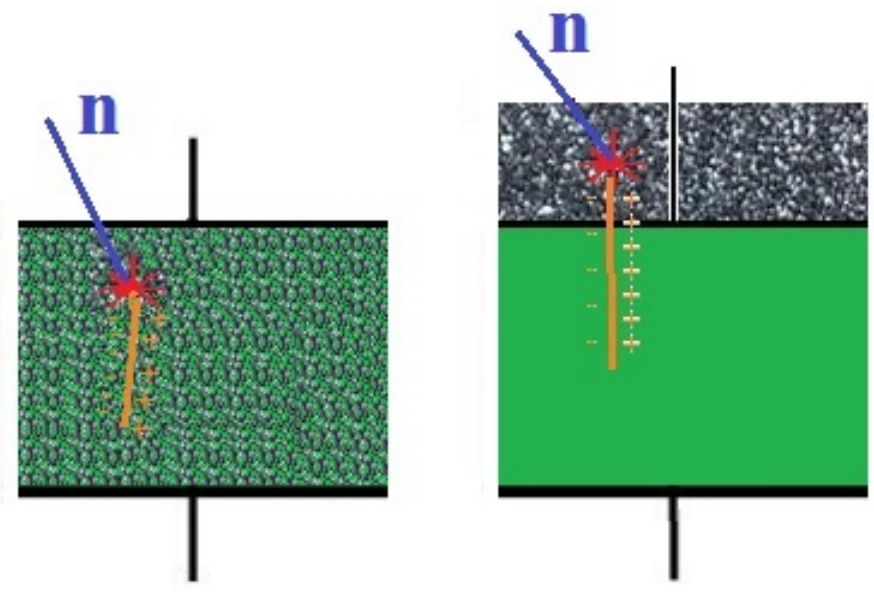

Figure 1: The two different approaches to solid state neutron detectors: reactive material detector and thin film converter deposition on semiconductor sensor.

can be obtained by using micro-machining technologies to obtain three dimensional geometries. By doing so, the aspect ratio between area and volume is improved with a consequent increase of the probability for reaction products to be detected. The key aspects are the optimization of the size of the cavities and of the gap regions in between them. Moreover, the deposition techniques of converter materials should be optimized to ensure conformal filling of small cavities and good stability.

Figure 1 resumes the main geometries proposed using ${ }^{6} \mathrm{Li}$ based neutron converter by Kansas State University (USA) [7][8][9] [10]. The best efficiency obtained by perforated neutron detector filled by ${ }^{6} \mathrm{Li}$ was of $29 \%$ at $10 \mathrm{~V}$ [11]. Also for these structures it was possible to use back to back stacking, leading to a reported efficiency of $42 \%$ at $3 \mathrm{~V}$ reverse bias [8].

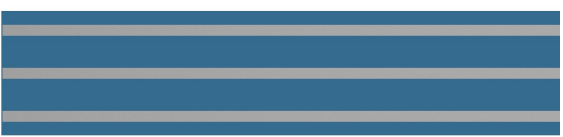

a) Linear trench

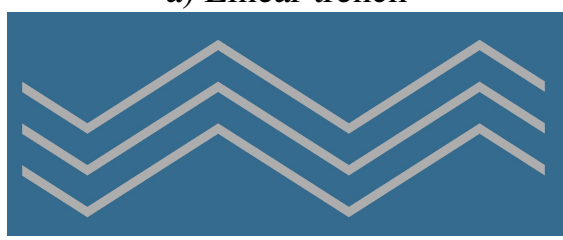

c) Chevron-type trench

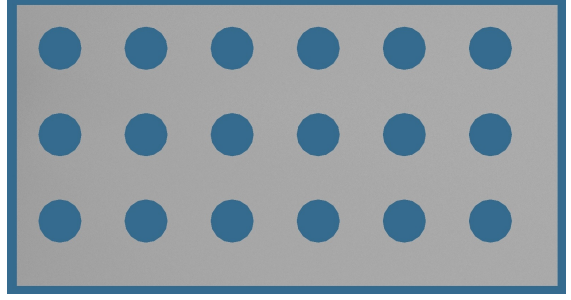

b) perforated sensor

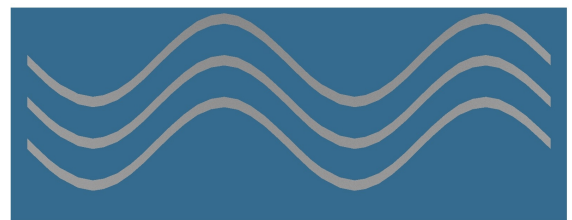

d) Sinusoidal trench

Figure 2: Three-dimensional neutron detector structures proposed by Kansas State University. 
Using 3D technology a different approach was followed by the Lawrence Livermore National Laboratory (USA). The geometry in this case is composed by silicon pillars of few micron of diameter surrounded by ${ }^{10} B$ converter [12][13][14]. Depending on the achievable pillar height it is possible to obtain very good results. A sketch of this sensor is reported in Fig.3. The efficiency ranges from $22 \%$ for $26 \mu \mathrm{m}$ high pillars to $48.5 \%$ for $50 \mu \mathrm{m}$ high pillars, with a a low level discrimination set to $30 \mathrm{keV}$ at $0 \mathrm{~V}$ bias [12].

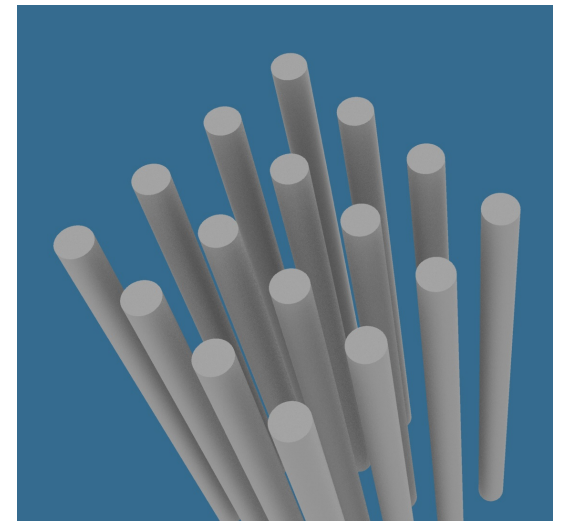

Figure 3: Pillar detector concept proposed by Lawrence Livermore National Laboratory.

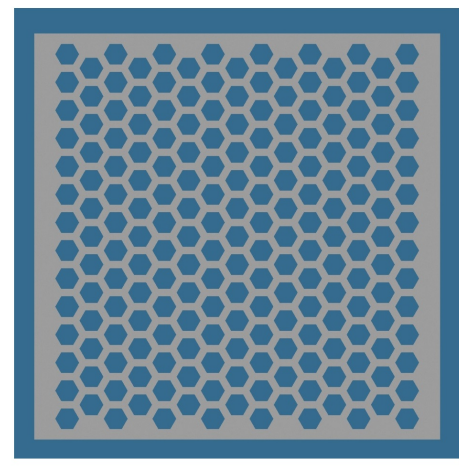

Figure 4: Sketch of honeycomb device proposed by Rensselaer Polytechnic Institute

Similar perforated structures with ${ }^{10} B$ converter have been reported by the Rensselaer Polytechnic Institute (USA) [15][16][17]. A sketch of the device, having a honeycomb structure, is reported in Fig. 4. The efficiency result was pretty good (26\%).

Some research groups are also active in Europe. Arrays of micro-machined pixels used to encapsulate crystals of a neutron sensitive scintillator material were fabricated at Delft University (Holland). The emitted light at $600 \mathrm{~nm}$ wavelength was used for the detection of neutrons[18]. The Czech Technical University in Prague (Czech Republic) reported thermal neutron test results of detectors fabricated with pyramidal dips on the surface and covered with ${ }^{6} \mathrm{LiF}$ [19]. The reported efficiency was of $6.3 \%$ for a single layer compared the $4.90 \%$ obtained with a reference planar detector. Among other interesting devices based on micro-machining are ultra thin 3D sensors with a planar converter coating $\left({ }^{10} B_{4} C\right)$ proposed by CNM Barcelona (Spain) to minimize the $\gamma$ ray sensitivity [20][21][22]. Moreover, SINTEF (Oslo, Norway) developed sensors with pyramidal grooves with a relative efficiency of up to $38 \%$ higher compared to planar[23]. The converter materials used were $T i B_{2}$ and ${ }^{6} \mathrm{LiF}$.

Starting from our experience with 3D detectors for High Energy Physics [24] we started the HYDE (HYbrid DEtectors of neutrons) project, aimed at the development of pixellated hybrid neutron detectors compatible with the read-out chips of the MEDIPIX family [25]. The first HYDE batch had the purpose to create a geometry that could be easily filled with different converter materials: Polysiloxane (for fast neutrons), ${ }^{10} \mathrm{~B}_{4} \mathrm{C},{ }^{6} \mathrm{LiF}$ and ${ }^{10} \mathrm{~B}$ (for thermal neutrons). The electrical and functional characterization have been reported in detail in [26][27][28], also in comparison to test results from planar sensors and Geant4 simulations. In the remaining part of this paper we will focus on the new detector we have designed: the geometry and technology will be described, and selected results from Geant4 and TCAD simulations will be reported. 


\section{HYDE2 device description}

The new detector design has the goal to improve the sensor performance and to solve some operational issues discovered during the experiments, while reducing the fabrication process complexity. As a starting material, Float Zone, P-type substrates are used, with a very high nominal resistivity in the range from 10 to $30 \mathrm{k} \Omega \cdot \mathrm{cm}$. The substrate thickness is $275 \mu \mathrm{m}$, due to the difficult procurement of thin wafers of 6-inch diameter, but ideally in the future thinner substrates will be used.

Devices consist of array of pixels with size of $55 \times 55 \mu \mathrm{m}^{2}$ so as to be compatible with the Medipix2 read-out chip. The ohmic side of each pixel is etched using Deep Reactive Ion Etching (DRIE) in order to create a web-like structure (see Fig.5). The junction side is patterned and doped by Phosphorus diffusion to define the $\mathrm{n}^{+}$single pixels, which are isolated at the surface by a $\mathrm{p}$ spray layer. On the opposite side (etched side) there is a $\mathrm{p}^{+}$diffusion to create an ohmic contact. It is important to note that such a layer is present only at the top of the walls that separate the etched trenches, thus allowing for a significantly reduction of the dead layer for reaction products at the trench walls. A schematic cross-section of the device is reported in Fig.6.

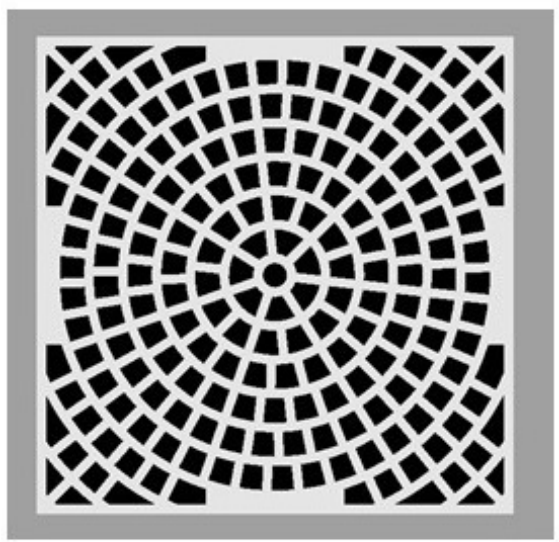

Figure 5: Rendering of the web-like structure.

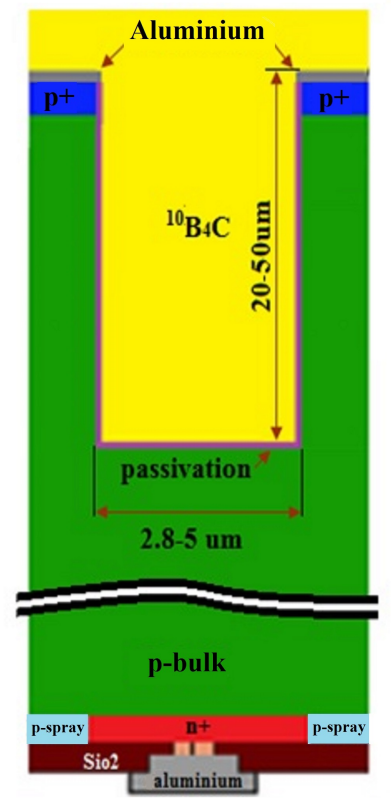

Figure 6: Schematic cross-section of a HYDE2 device (not to scale).

The most important design parameters are the lateral sizes of the trenches and of the silicon walls in between them, as well as the trench depth. Taking into account simulation results, the wall size was varied in the range from 1.1 to $3 \mu \mathrm{m}$, whereas the trench size was varied between 2 and $5 \mu \mathrm{m}$. The trench depth should be ideally $40 \mu \mathrm{m}$, but this value is in fact difficult to achieve. Many technological tests were performed to define the final fabrication process. The results are encouraging but suggest to stop the DRIE etching of the trenches to a depth of about $25 \mu \mathrm{m}$ in order to avoid mechanical and thermal problems (the heat produced during etching is difficult to be removed due to the narrow walls around the trenches). 
An optical micrograph of two adjacent pixels from the etched side is shown in Fig.7, whereas a Scanning Electron Microscope (SEM) picture of a cross section is shown in Fig. 8. The quality of the etched trenches is still good enough at $25 \mu \mathrm{m}$ depth.

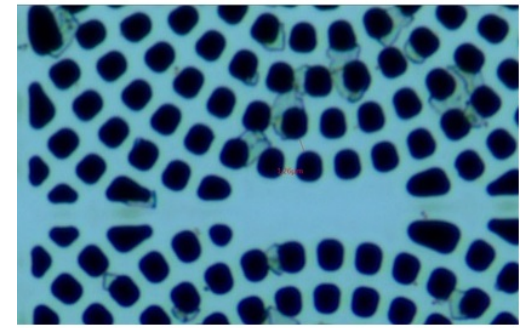

Figure 7: Optical micrograph of two pixels from the etched side of a HYDE2 device.

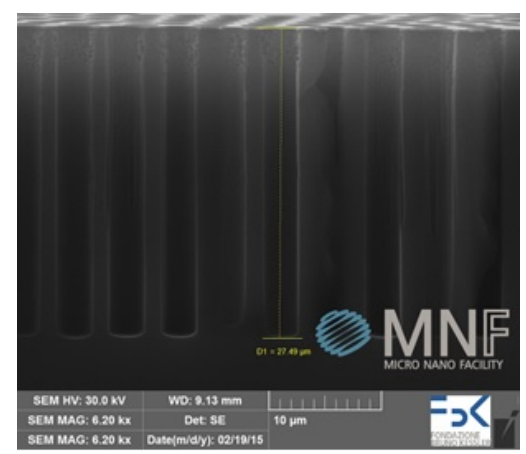

Figure 8: SEM picture of the cross section of a HYDE2 device.

The cross section of the sensor also highlights the presence of a layer of passivation deposited on the etched side. It is obtained by a ALD (Atomic Layer Deposition) of $\mathrm{Al}_{2} \mathrm{O}_{3}$ (also known as alumina). It allows to have an electrical insulation between the converter material and silicon, as well as to passivate the silicon surface. This layer is indeed very thin (tens of nm ) so as to cause a minor energy loss for the reaction products. Alumina is characterized by a relatively high density of negative charge at the interface, that might affect the electrical behaviour of the sensor. The simulation section discusses in detail this aspect.

\section{Geant4 Simulations}

Simulations using the Geant 4 code were performed to accurately model the interaction of particles with matter. Thanks to the different toolkits present on the kernel, it was possible to import the geometry directly from the layout [29], and to define the physics to be used and running the simulations of different events using the defined environment [30][31]. The physics section was defined using a pre-existent model called QGSP_BERT_HP using the neutron cross section contained in the GDML library. The simulated geometry is a single pixel as previously described filled with converter material. The trenches have the nominal design depth of $40 \mu \mathrm{m}$. The composition of the considered converter materials are ${ }^{10} B$ with a density of $2.46 \mathrm{~g} / \mathrm{cm}^{3}$ and ${ }^{10} B_{4} C$ with a density of $2.520 \mathrm{~g} / \mathrm{cm}^{3}$. Figure 9 shows the simulated energy spectra for 10 millions of events for the best device geometry.

The simulated absolute efficiency, defined as the ratio of recorded events from $\alpha$ and ${ }^{7} \mathrm{Li}$ causing energy release within the silicon volume and the total number of impinging neutrons is about $18 \%$ for ${ }^{10} \mathrm{~B}_{4} \mathrm{C}$ and $19 \%$ for ${ }^{10} \mathrm{~B}$. Three of the four energy peaks coming from the reactions are visible in the spectra: the $\mathrm{Li}$ at $0.84 \mathrm{MeV}$ and the alpha particles at 1.47 and $1.78 \mathrm{MeV}$. The low probability of generation of $\mathrm{Li}$ at $1.01 \mathrm{MeV}$ makes the related peak indistinguishable in the spectra. With 3D geometry it is possible to capture both particles from a reaction at the same time. This is the reason why the spectra are extended to energies higher than $1.78 \mathrm{MeV}$. In particular the 


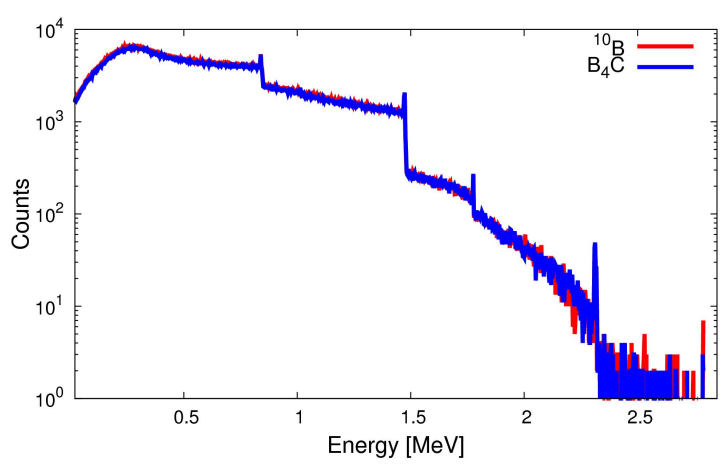

Figure 9: Geant 4 energy spectra for a pixel simulated with ${ }^{10} B_{4} C$ and ${ }^{10} B$ converter.

most probable reaction gives a peak also in this part of the spectra at around $2.3 \mathrm{MeV}$ as a sum of alpha and Li energy.

\section{TCAD simulations}

Device simulations were performed with Synopsys TCAD software. The aim of this study was to estimate the electrical characteristics and the charge collection properties of the sensor in the presence of high densities of generated electron-hole pairs. The impact of the fixed negative charge of the alumina passivation layer was also investigated. The simulation domain is a cross section comprising half trench-wall-half trench in two dimensions. This choice was necessary due to the geometry of the single pixel, that would make simulations in three dimensions practically impossible due to discretization grid complexity. Since the software does not include alumina among available materials, in the simulations the passivation layer was modelled as silicon oxide with negative fixed charge density. The first set of simulations regarded the electric field distribution in the sensors and the effect of the recombination at the surface of etched trenches on the reverse current. Since an accurate estimate of the surface recombination velocity $\left(S_{0}\right)$ after a DRIE step is very difficult to obtain, it was chosen to vary the value of this parameter over a wide interval, from 0 to $1000 \mathrm{~cm} / \mathrm{s}$. On the contrary, the charge density for the alumina layer $\left(N_{o x}\right)$ was fixed to $-10^{12}$ $\mathrm{cm}^{-2}$ [32]. Similar results were in fact observed using different charge concentration values. A comparison between passivated and non passivated trenches has been considered, and it will also be carried out as a process split in the device fabrication.

Figure 10 shows the reverse current-voltage curves for different values of the surface recombination velocity. The huge difference between the passivated and the non passivated device is evident. In the non passivated device, the current is significantly increased as the surface recombination velocity is increased. On the contrary, in the passivated device the presence of high density of negative charge at the interface completely suppresses the surface recombination effect.

In order to explain the reason for this big difference, it is useful to investigate the distribution of the electric field inside the most critical region in the sensor, i.e., the wall between the trenches (see Fig.11). In case passivation is not present (Fig.11a) the absence of any fixed charge causes a large electric field in this region, that is fully depleted. As a result, surface recombination significantly 


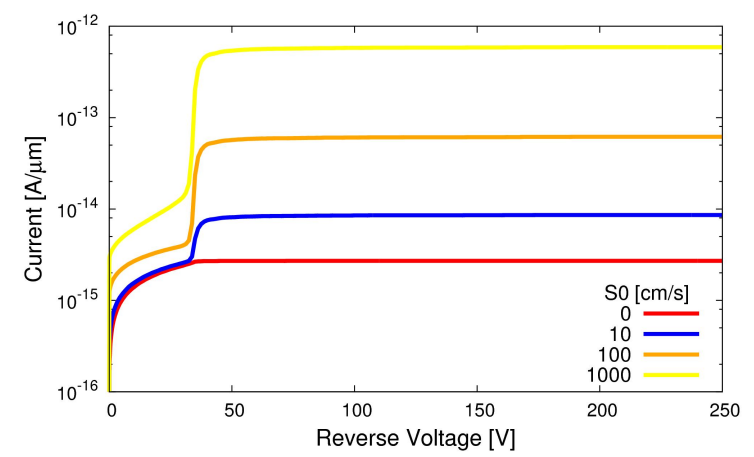

a) without $\mathrm{Al}_{2} \mathrm{O}_{3}$

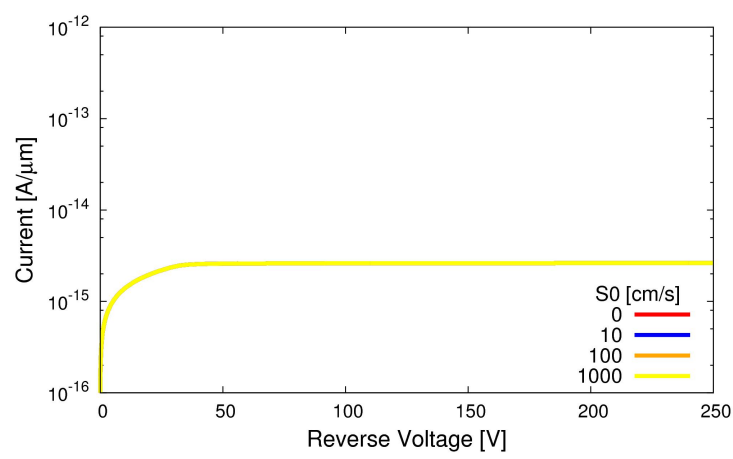

b) with $\mathrm{Al}_{2} \mathrm{O}_{3}\left(N_{o x}=-10^{12} \mathrm{~cm}^{-2}\right)$

Figure 10: Simulated I-V curves for different surface recombination velocities.

contributes to the leakage current that reaches high values with impact on the noise. The situation is different in the presence of passivation with negative fixed charge (Fig.11b): in this case the region of interest is not fully depleted and the electric field is weak, so that the surface recombination does not contribute to the leakage current. On one hand, this is a positive feature since it decreases noise, but it also implies that the generated charge should be collected by diffusion within this region, delaying the signal and leading to possible charge losses.

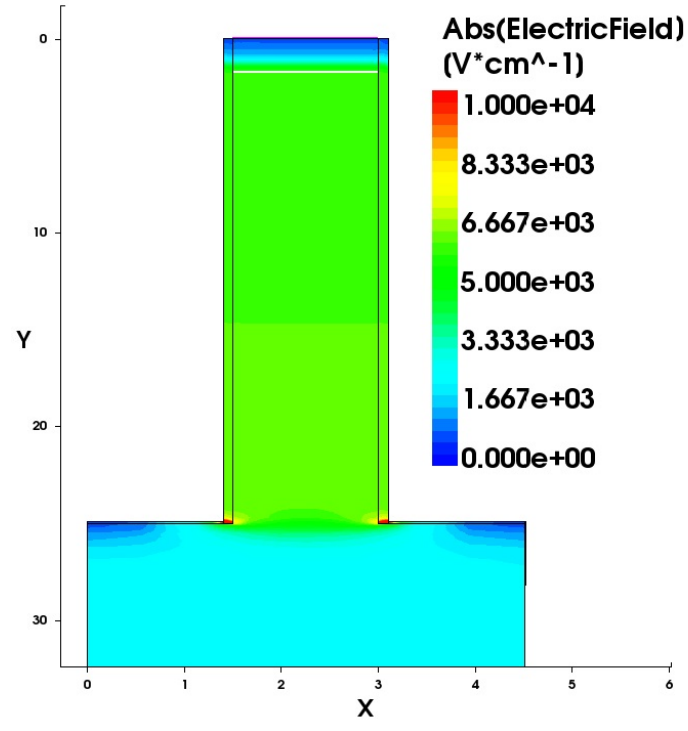

a) without $\mathrm{Al}_{2} \mathrm{O}_{3}$

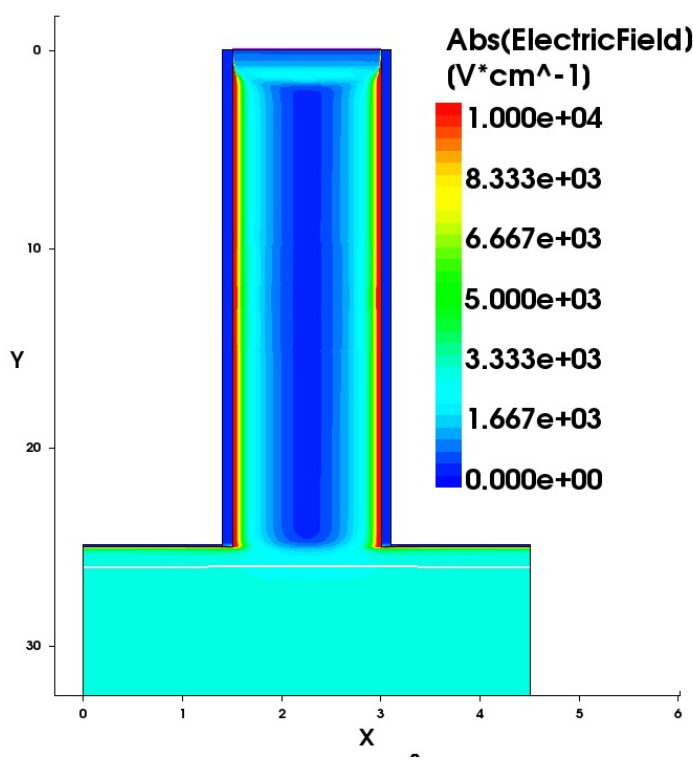

b) with $\mathrm{Al}_{2} \mathrm{O}_{3}\left(\mathrm{~N}_{o x}=-10^{12} \mathrm{~cm}^{-2}\right)$

Figure 11: Simulated distribution of the electric field in the critical region of a HYDE2 device at $100 \mathrm{~V}$ reverse bias.

Charge collection efficiency was a major issue in the first prototypes of HYDE sensors, mainly because the collection time was very long, in the order of tens of $\mu$ s [26]. In this new sensors the goal is to achieve a full charge collection in a few $\mu$ s only. To gain insight into this aspect, further simulations were performed maintaining the same simulation domain with particles hitting 
perpendicularly to the surface at the center of the wall between the trenches (worst case condition). The reaction products are modelled as heavy ions with a parametrized release of energy and a short range: the corresponding charge was varied from 80 electron/hole pairs $/ \mu \mathrm{m}$ to $3.75 \times 10^{5}$ electron/hole pairs $/ \mu \mathrm{m}$. This wide range of values was chosen in order to cover the release of charge from the level of a m.i.p to that of an alpha particle at the most probable energy value of 1.47 MeV. The integration time of the current signal was purposely limited to only $2 \mu \mathrm{s}$.

Simulation results are summarized in Fig. 12, showing the charge collection efficiency (CCE) as a function of the signal charge at different values of surface recombination velocity. In the absence of passivation, hence without interface charge (Fig. 12a), surface recombination effects are important at high signal charge and cause a significant decrease of the CCE. It should be noted that at high signal charge plasma effects are also present [33], but their impact at low $S_{0}$ is limited. The presence of alumina passivation and related negative charge density has a beneficial impact on the charge collection efficiency (Fig. 12b) by strongly attenuating surface recombination effects, so that a CCE decrease of only a few $\%$ at high signal charge is observed, due to plasma effects alone. Simulations were also repeated at $N_{o x}=-10^{11} \mathrm{~cm}^{-2}$ (not shown): in this case the impact of surface recombination is not negligible, with a worst case CCE loss of $20 \%$ at the maximum signal charge for $S_{0}=1000 \mathrm{~cm} / \mathrm{s}$.

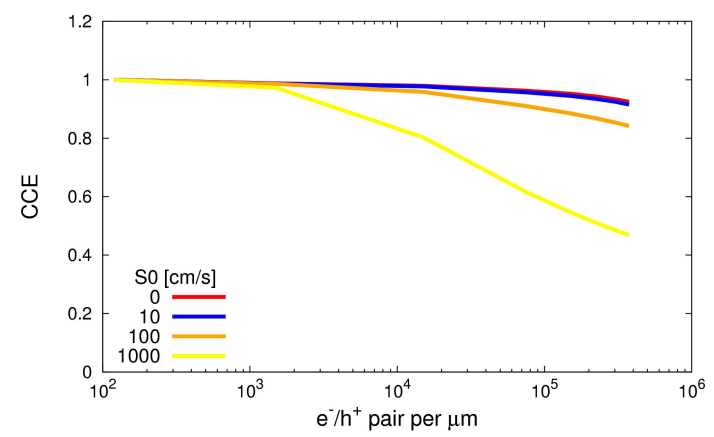

a) without $\mathrm{Al}_{2} \mathrm{O}_{3}$

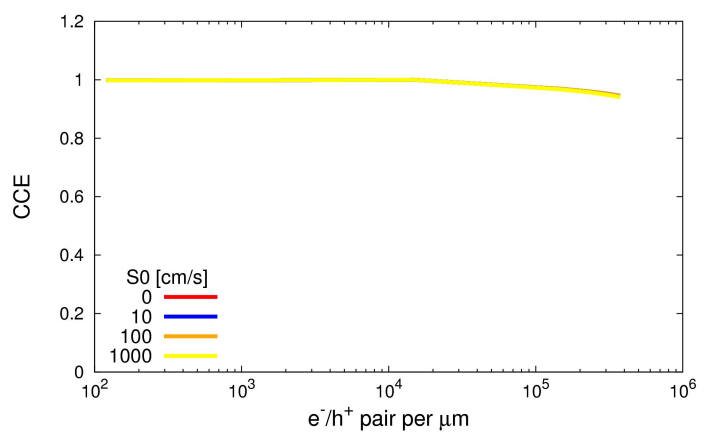

b) with $\mathrm{Al}_{2} \mathrm{O}_{3}\left(\mathrm{~N}_{\mathrm{ox}}=-10^{12} \mathrm{~cm}^{-2}\right)$

Figure 12: Simulated charge collection efficiency as a function of the signal charge at different values of surface recombination velocity.

\section{Conclusion}

In the past few years, pushed by the ${ }^{3} \mathrm{He}$ shortage and the increasing demand in several applications, in particular homeland security, important progress has been made in semiconductor neutron detectors. In this paper, we have reviewed the most interesting silicon based approaches, that exploit micro-machining techniques to obtain three-dimensional structures, able to reach relatively high detection efficiency, up to $\sim 50 \%$.

We have also reported some recent results from our R\&D activity in the INFN HYDE project. Based on the encouraging results from first prototypes, we have designed a new sensor geometry, suitable for thermal neutron imaging (i.e., by coupling to a MEDIPIX2 read-out chip). Geant4 and TCAD simulations allowed to validate the sensor concept and estimate its performance, including 
detection efficiency in the order of $20 \%$ and maximum rate in the order of $1 \mathrm{MHz}$. A batch of these sensors is being fabricated at FBK and will be available for testing soon.

\section{References}

[1] D. S. McGregor et al., Present status of microstructured semiconductor neutron detectors, Journal of Crystal Growth 379 (2013) 99.

[2] A. N. Caruso, The physics of solid-state neutron detector materials and geometries, J. Phys.: Condens. Matter 22 (2010) 443201.

[3] D. Emin et al., A proposed boron-carbide-based solid-state neutron detector, J. Appl. Phys. 013529 (2005) 97.

[4] E. Tupitsyn et al., Single crystal of LiInSe 2 semiconductor for neutron detector, Appl. Phys. Letter 101 (2012) 202101.

[5] D. S. McGregor et al., Designs for Thin Film Coated Semiconductor Thermal Neutron Detectors, IEEE Nuclear Science Symposium Conference Record 14 (2001) 2454.

[6] D. S. McGregor et al., Thin-film-coated bulk GaAs detectors for thermal and fast neutron measurements, Nucl. Instrum. Methods Phys. Res. A 466 (2001) 126.

[7] S.L. Bellinger et al., Enhanced variant designs and characteristics of the microstructured solid-state neutron detector, Nucl. Instrum. Methods Phys. Res. A 652 (2011) 387.

[8] S.L. Bellinger et al., Improved High Efficiency Stacked Microstructured Neutron Detectors Back filled With Nanoparticle LiF, IEEE Trans. Nucl. Sci. 59 (2012) 167.

[9] C.J. Solomon et al. Reduced efficiency variation in perforated neutron detectors with sinusoidal trench design, Nucl. Instrum. Methods Phys. Res. A 618 (2010) 260.

[10] S.L. Bellinger et al., High-efficiency microstructured semiconductor neutron detectors that are arrayed, dual-integrated, and stacked, Applied Radiation and Isotopes 70 (2012) 1121.

[11] D. S. McGregor et al., Micro-structured high-efficiency semiconductor neutron detectors, IEEE Nuclear Science Symposium, Conference Record (2008) 446.

[12] Q. Shao et al., High aspect ratio composite structures with $48.5 \%$ thermal neutron detection efficiency, Applied Physics Letters 102 (2010) 063505.

[13] R. Nikolic et al., Silicon-based, pillar-structured thermal-neutron detectors, SPIE Newsroom (2010) doi:10.1117/2.1201009.003115

[14] R. Nikolic et al., Roadmap for High Efficiency Solid-State Neutron Detectors, Proceedings of SPIE 6013 (2005) 601305.

[15] R. Dahal et al., Self-powered micro-structured solid state neutron detector with very low leakage current and high efficiency, Applied Physics Letters 100 (2012) 243507.

[16] Y. Danon et al., Towards high efficiency solid-state thermal and fast neutron detectors, JINST 7 (2012) C03014.

[17] K. C. Huang et al., Boron filling of high aspect ratio holes by chemical vapor deposition for solid-state neutron detector applications, J. Vac. Sci. Technol. B 30 (2012) 051204.

[18] C.P. Allier et al., Thin Photodiodes for a Neutron Scintillator Silicon-Well Detector, IEEE Trans. Nucl. Sci. 48 (2001) 1154. 
[19] J. Uher et al., Characterization of 3D thermal neutron semiconductor detectors, Nucl. Instrum. Methods Phys. Res. A, 576 (2007) 32.

[20] C. Guardiola et al. Ultra-thin 3D silicon sensors for neutron detection, JINST 7 (2012) P03006.

[21] C. Fleta et al. Fabrication and nuclear reactor tests of ultra-thin 3D silicon neutron detectors with a boron carbide converter, JINST 9 (2014) P04010.

[22] C. Guardiola et al. Neutron measurements with ultra-thin 3D silicon sensors in a radiotherapy treatment room using a Siemens PRIMUS linac, Phys. Med. Biol. 58 (2013) 3227.

[23] A. Kok et al, Silicon sensors with pyramidal structures for neutron imaging, JINST 9 (2014) C04011.

[24] G. Giacomini et al, Development of Double-Sided Full-Passing-Column 3D Sensors at FBK, IEEE Trans. Nucl. Sci. 60 (2013) 2357.

[25] http://medipix.web.cern.ch/medipix/

[26] R. Mendicino et al., Novel 3D silicon sensors for neutron detection, JINST 9 (2014) C05001.

[27] G. -F. Dalla Betta et al., Hybrid detectors of neutrons based on 3D silicon sensors with PolySiloxane converter, IEEE Room Temperature Semiconductors Conference, Conference Record (2013) R05-32.

[28] R. Mendicino et al., Characterization of 3D and planar Si diodes with different neutron converter materials, Nucl. Instrum. Methods Phys. Res. A 796 (2015) 23.

[29] C. M. Poole et al., A CAD Interface for GEANT4, Australasian Physical \& Engineering Science in Medicine 35 (2012) 329.

[30] S. Agostinelli et al., Geant4a simulation toolkit, Nucl. Instrum. Methods Phys. Res. A 506 (2003) 250.

[31] J Allison et al., Geant4 Developments and Applications. Physics, Developments and Applications. Physics 53 (2006) 270.

[32] G. Agostinelli et al., Very low surface recombination velocities on p-type silicon wafer passivated with a dielectric with fixed negative charge, Solar Energy Material \& Cells 90 (2006) 3438.

[33] J. Becker et al., Simulation and experimental study of plasma effects in planar silicon sensors Nucl. Instrum. Methods Phys. Res. A 624 (2010) 716. 